\title{
Search for a correlation between the UHECRs measured by the Pierre Auger Observatory and the Telescope Array and the neutrino candidate events from IceCube
}

The IceCube Collaboration ${ }^{1}$, The Pierre Auger Collaboration ${ }^{2}$, The Telescope Array Collaboration ${ }^{3}$

${ }^{1}$ http://icecube.wisc.edu/collaboration/authors/icrc15_icecube

${ }^{2}$ http://www.auger.org/archive/authors_2015_ICRC.html

${ }^{3}$ http://www.telescopearray.org/index.php/research/collaborators

\section{E-mail: christovecern.ch}

We have conducted three searches for correlations between ultra-high energy cosmic rays detected by the Telescope Array and the Pierre Auger Observatory, and high-energy neutrino candidate events from IceCube. Two cross-correlation analyses with UHECRs are done: one with 39 cascades from the IceCube 'high-energy starting events' sample and the other with 16 high-energy 'track events'. The angular separation between the arrival directions of neutrinos and UHECRs is scanned over. The same events are also used in a separate search using a maximum likelihood approach, after the neutrino arrival directions are stacked. To estimate the significance we assume UHECR magnetic deflections to be inversely proportional to their energy, with values $3^{\circ}, 6^{\circ}$ and $9^{\circ}$ at $100 \mathrm{EeV}$ to allow for the uncertainties on the magnetic field strength and UHECR charge. A similar analysis is performed on stacked UHECR arrival directions and the IceCube sample of through-going muon track events which were optimized for neutrino point-source searches.

Corresponding authors: A. Christov ${ }^{4}$, G. Golup ${ }^{* 5}$, J. Aublin ${ }^{6}$, L. Caccianiga ${ }^{6}$, P.L. Ghia ${ }^{6}$, T. Montaruli $^{4}$, M. Rameez ${ }^{4}$, E. Roulet ${ }^{5}$, H. Sagawa ${ }^{7}$, P. Tinyakov ${ }^{8}$, M. Unger ${ }^{9}$

${ }^{4}$ Départment de physique nucléaire et corpusculaire, Université de Genève, 24 Quai Ernest Ansermet, 1211 Genève, Switzerland.

${ }^{5}$ Centro Atómico Bariloche, Av. Bustillo 9500, S. C. de Bariloche 8400, Argentina.

${ }^{6}$ Laboratoire de Physique Nucléaire et de Hautes Energies (LPNHE), Universités Paris 6 et Paris 7, CNRS-IN2P3, 4 place Jussieu, 75252, Paris, France.

${ }^{7}$ Institute for Cosmic Ray Research, University of Tokyo, Kashiwa, Chiba, Japan.

${ }^{8}$ Service de Physique Théorique, Université Libre de Bruxelles, Boulevard du Triomphe (Campus de la Plaine), Ixelles 1050, Belgium.

${ }^{9}$ Karlsruhe Institute of Technology - Campus North - Institut für Kernphysik, Karlsruhe, Germany and New York University, New York, USA.

The 34th International Cosmic Ray Conference,

30 July- 6 August, 2015

The Hague, The Netherlands

* Speaker. 


\section{Introduction}

A multi-messenger approach can help to identify the sources of ultra-high energy cosmic rays (UHECRs). It is difficult to do so from their arrival directions since CRs are charged particles so are deflected en-route to Earth. This deflection cannot be computed precisely since the CR composition at ultra-high energies as well as the intervening magnetic field strength are poorly known. If the CR composition is light i.e. mainly protons, the magnetic deflection may be only a few degrees above a few tens of EeV. Secondary particles including neutrinos $(v \mathrm{~s})$ are produced in the sources by the interactions between the CRs and ambient photon and matter fields. Neutrinos have no charge and interact only through the weak force, so their arrival directions do point back to where they originated from, although they are also hard to detect for the same reason. In this work we describe a joint analysis by the IceCube, Pierre Auger and Telescope Array Collaborations to search for angular correlations between the arrival directions of high-energy $v$ s and UHECRs that would provide insight into the long-standing open question of cosmic ray origin.

\section{The observatories and data sets}

\subsection{The IceCube Neutrino Telescope}

IceCube is a cubic-kilometer neutrino detector installed in the ice at the geographic South Pole [1] between depths of $1450 \mathrm{~m}$ and $2450 \mathrm{~m}$. Neutrino reconstruction relies on the optical detection of Cherenkov radiation emitted by secondary particles produced in $v$ interactions in the surrounding ice or the nearby bedrock.

Depending on the flavor of the interacting neutrino and the type of interaction, different signatures are expected in the detector. The one with the best angular resolution $\left(\sim 1^{\circ}\right)$ is the charged current $v_{\mu}$ interaction where a track is produced as the outgoing muon traverses the detector. Cascades are produced in the detector as a result of charged current $v_{e, \tau}$ interactions or all neutral current neutrino interactions. In this case the angular resolution is poorer (around $15^{\circ}$ above 100 $\mathrm{TeV}$ ). The resolution of the deposited energy for tracks and cascades is around 15\% [2] but cascades have a better resolution for the reconstructed neutrino energy since most of the energy is deposited in the detector, which is not the case for tracks.

Different data sets are considered in this work. A set of cascades that have been detected in a search for high-energy events where the interaction occurs within the detector is used [3]. This set of 39 cascades, which is part of the HESE ('High-Energy Starting Events') set, consists of data taken between May 2010 and May 2014 and is called 'high-energy cascades' in the following (deposited energy range: $\sim 30-2000 \mathrm{TeV}$ ). A second set of events referred to as 'high-energy tracks' (energy above $\sim 70 \mathrm{TeV}$ ) is formed by two parts. The first part is the 7 tracks in the HESE sample [3] that have energies and directions which make them more likely to be of extraterrestrial origin than the other track events in that sample. The second part is 9 muon tracks found in a search of a diffuse up-going $v_{\mu}$ flux [4]. These 9 muon tracks, found in two years of data (May 2010-May 2012), belong to a high energy-proxy excess with respect to atmospheric predictions. This excess is compatible with an astrophysical $E^{-2}$ flux at the level indicated by the HESE analysis [4].

The third data set used is called '4-year point-source sample' [5] and consists of events with sub-degree median angular resolution detected between May 2008 and May 2012. The set includes 
about 400,000 events, mostly up-going atmospheric $v$ s from the Northern hemisphere and highenergy atmospheric muons from the Southern hemisphere.

\subsection{The Pierre Auger Observatory}

The Pierre Auger Observatory is located in Malargüe, Argentina $\left(35.2^{\circ} \mathrm{S}, 69.5^{\circ} \mathrm{W}, 1400 \mathrm{~m}\right.$ a.s.1.) [6]. It consists of a surface array of 1660 water-Cherenkov detectors covering an area of approximately $3000 \mathrm{~km}^{2}$. The array is overlooked by 27 telescopes at four sites which constitute the fluorescence detector. The surface and air fluorescence detectors are designed to perform complementary measurements of air showers created by UHECRs.

The data set used for the present analysis includes 231 events with $E>52 \mathrm{EeV}$ and zenith angles smaller than $80^{\circ}$ recorded by the surface detector array from January 2004 to March 2014 [7]. The exposure determined by geometrical considerations for the period analyzed amounts to $66,452 \mathrm{~km}^{2} \mathrm{sr} y \mathrm{y}$. The angular resolution, defined as the 68 th percentile of the distribution of opening angles between the true and reconstructed directions of simulated events, is better than $0.9^{\circ}$ [8]. The absolute energy scale, given by the fluorescence calibration, has a systematic uncertainty of $14 \%$ and the energy resolution is about $12 \%$ [9].

\subsection{Telescope Array}

The Telescope Array (TA) is located in Utah, USA (39. $3^{\circ} \mathrm{N}, 112.9^{\circ} \mathrm{W}, 1400 \mathrm{~m}$ a.s.1.) [10] and detects extensive air showers generated by UHECRs. It comprises a $700 \mathrm{~km}^{2}$ surface array of 507 plastic scintillation detectors, $3 \mathrm{~m}^{2}$ each, distributed in a square grid with $1.2 \mathrm{~km}$ spacing. The array is overlooked by 3 fluorescence detector stations with 38 telescopes.

The UHECR sample considered in the present analysis consists of 87 events with $E>57 \mathrm{EeV}$ and zenith angles smaller than $55^{\circ}$ collected between May 2008 and May 2014 by the surface detector. A subset of events has been published in [11]. The total exposure is around $9,500 \mathrm{~km}^{2} \mathrm{sr} \mathrm{yr}$. The angular resolution is better than $1.5^{\circ}$. The energy scale of the surface detector is also calibrated with the fluorescence detector. The energy resolution is better than $20 \%$ with a systematic uncertainty on the absolute energy scale of $21 \%$ [12].

\section{Data analyses}

There are three different analyses which are presented in detail in this Section. A crosscorrelation and a stacking likelihood analysis are done on the sample of high-energy cascades and high-energy tracks and the UHECRs detected by Auger and TA. Cascade and track-like events are considered separately since, due to their different angular resolutions, the angular distance at which a signal (if any) can be detected would be different. A third analysis is performed on stacked UHECRs and the IceCube 4-year point-source sample.

The magnetic deflections of CRs have to be accounted for in the likelihood tests. For simplicity, we model individual deflections as a random variable 2-dimensional Gaussian distribution with the energy-dependent standard deviation $\sigma_{\mathrm{MD}}\left(E_{\mathrm{CR}}\right)=D \times 100 \mathrm{EeV} / E_{\mathrm{CR}}$, and we consider the representative values $D=3^{\circ}, 6^{\circ}$ and $9^{\circ}$ (the latter is just used for the likelihood test with the high-energy cascades and high-energy tracks). These values are reasonable test values as shown by a backtracking simulation of the detected UHECRs in the galactic magnetic field models of 


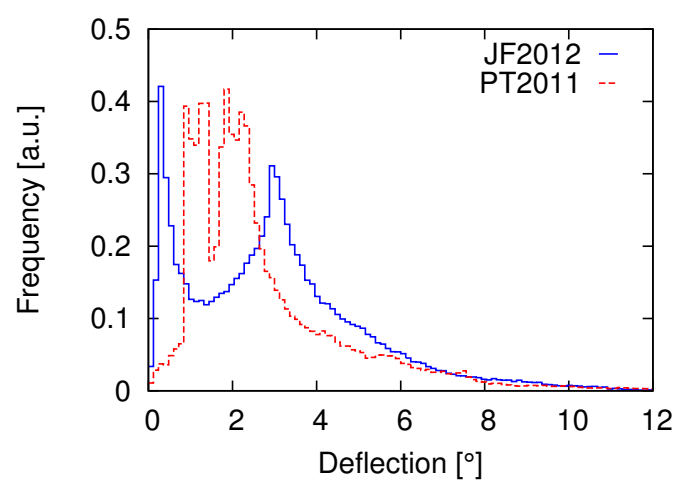

Figure 1: Distribution of UHECR deflections in two models for the regular component of the galactic magnetic field, PT2011 [13] and JF2012 [14], for a rigidity $E / Z=100 \mathrm{EeV}$.

Pshirkov et al. [13] and Jansson and Farrar [14] and assuming these are protons with $E=100 \mathrm{EeV}$. The distributions of the obtained deflections are different for each model (Fig. 1), but the median values for both are $2.7^{\circ}$. We have then chosen an average value of $3^{\circ}$. The values of $6^{\circ}$ and $9^{\circ}$ are also considered to account for larger deflections that could arise from other light CR components $(Z=2,3)$ or a stronger than predicted strength of the intervening magnetic fields.

\subsection{UHECR correlation analyses with high-energy cascades and high-energy tracks}

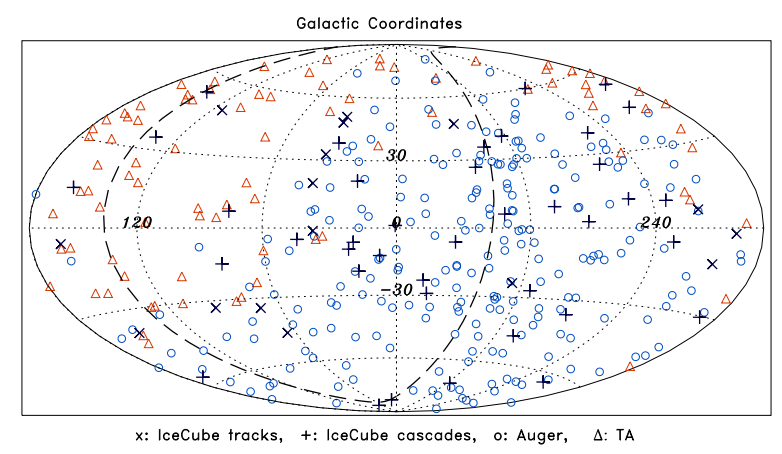

Figure 2: Aitoff-Hammer projection of the sky in galactic coordinates showing the arrival directions of the IceCube high-energy cascades (plus signs) and high-energy tracks (crosses), and the UHECRs detected by Auger (circles) and TA (triangles). The dashed line indicates the Super-galactic plane.

The arrival directions of the high-energy tracks and high-energy cascades in IceCube, and of the UHECRs measured by Auger and TA are shown in Fig. 2 in galactic coordinates. Two different analyses are performed with this data set: a cross-correlation and a stacking likelihood analysis.

The cross-correlation method consists of computing the number of UHECR- $v$ pairs as a function of their angular separation $\alpha, n_{\mathrm{p}}(\alpha)$, and comparing it to the expectation from an isotropic distribution of arrival directions of CRs. The angular scan performed in this case is between $1^{\circ}$ and $30^{\circ}$ with a step of $1^{\circ}$ and, due to this scan, the method does not rely on any assumption about the exact value of the strength of the magnetic deflections, unlike the likelihood method.

In Fig. 3 we show the results obtained applying the cross-correlation method to the data. For the case of the sample of high-energy tracks, the maximum departure from the isotropic expectation 
of CRs (fixing the positions of the $v \mathrm{~s}$ ) obtained is at an angular distance of $2^{\circ}$, where 1.5 pairs were expected on average and 4 pairs were detected. The post-trial $p$-value is $34 \%$. For the analysis done using the high-energy cascade events, the smallest pre-trial $p$-value occurs at an angular distance of $22^{\circ}$, for which 575 pairs are observed while 490.3 were expected on average. The post-trial $p$-value is $5 \times 10^{-4}$ with respect to expectations of an isotropic flux of CRs. As an a posteriori study, we also evaluated the significance under the hypothesis of an isotropic distribution of neutrinos, fixing the UHECR arrival directions (note that this alternative hypothesis preserves the degree of anisotropy in the arrival directions of CRs that is suggested by the TA 'hot spot' [11] or the excess around Cen A reported by Auger [7]). The post-trial $p$-value is $8.5 \times 10^{-3}$. Thus the cross-correlation of UHECRs with the high-energy cascades provides a potentially interesting result, which we will continue to monitor in the future.
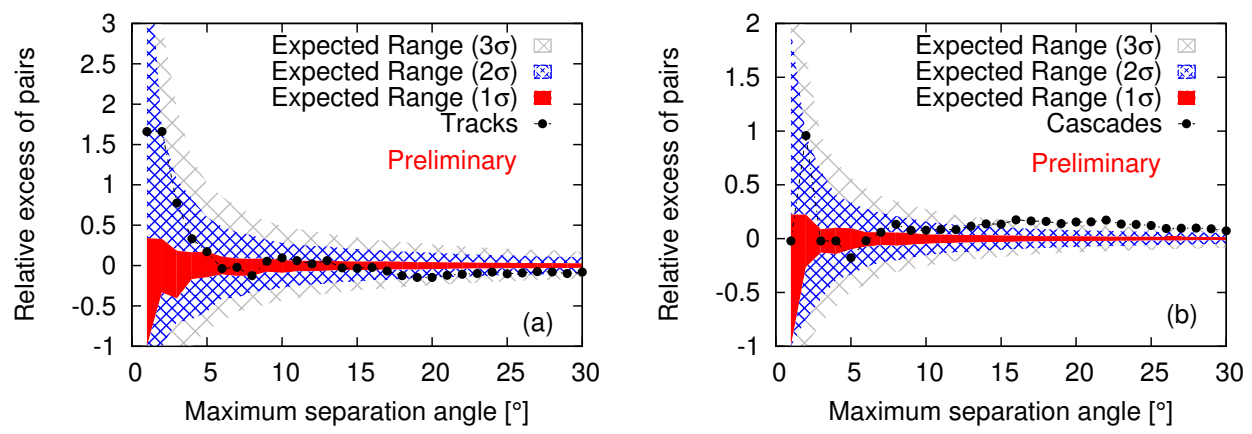

Figure 3: Relative excess of pairs, $\left[n_{\mathrm{p}}(\alpha) /\left\langle n_{\mathrm{p}}^{\text {iso }}(\alpha)\right\rangle\right]-1$, as a function of the maximum angular separation between the neutrino and UHECR pairs, for the analysis done with the high-energy tracks (a) and with the high-energy cascades (b). The $1 \sigma, 2 \sigma$ and $3 \sigma$ fluctuations expected from an isotropic distribution of arrival directions of CRs are shown in red, blue and grey, respectively.

Stacking a set of sources is a well known way of accumulating multiple weaker signals to enhance the discovery potential. Since $v$ s are not deflected on their way to Earth, the stacking over sources is replaced by stacking over the set of $v$ arrival directions. An unbinned likelihood method is used [15], with the log of the likelihood function defined as:

$$
\ln \mathscr{L}\left(n_{\mathrm{s}}\right)=\sum_{i=1}^{N_{\text {Auger }}} \ln \left(\frac{n_{\mathrm{s}}}{N_{\mathrm{CR}}} S_{\text {Auger }}^{i}+\frac{N_{\mathrm{CR}}-n_{\mathrm{s}}}{N_{\mathrm{CR}}} B_{\text {Auger }}^{i}\right)+\sum_{i=1}^{N_{\mathrm{TA}}} \ln \left(\frac{n_{\mathrm{s}}}{N_{\mathrm{CR}}} S_{\mathrm{TA}}^{i}+\frac{N_{\mathrm{CR}}-n_{\mathrm{s}}}{N_{\mathrm{CR}}} B_{\mathrm{TA}}^{i}\right),
$$

where $n_{\mathrm{s}}$, the number of signal events, is the only free parameter, $N_{\mathrm{CR}}=N_{\text {Auger }}+N_{\mathrm{TA}}, S_{\text {Auger }}^{i}$ and $S_{\mathrm{TA}}^{i}$ are the signal PDFs (Probability Distribution Functions) for Auger and for TA, respectively, and $B_{\text {Auger }}^{i}$ and $B_{\mathrm{TA}}^{i}$ are the corresponding background PDFs. The signal PDFs, in which the different neutrino positions are stacked, take into account the exposure and angular resolution of the CR observatories, the assumed CR magnetic deflections and the likelihood maps for the reconstruction of the $v$ s arrival directions (Fig. 4). The background PDFs are the normalized exposures of the CR observatories. The test statistic $T S$ is defined as: $T S=-2 \ln \frac{\mathscr{L}\left(n_{\mathrm{s}}\right)}{\mathscr{L}\left(n_{\mathrm{s}}=0\right)}$ and follows a distribution close to $\chi^{2}$ with one degree of freedom.

The results for the stacking method are shown in Table 1. The most significant deviation from an isotropic flux of CRs occurs for the magnetic deflection parameter $D=6^{\circ}$ with the high-energy 


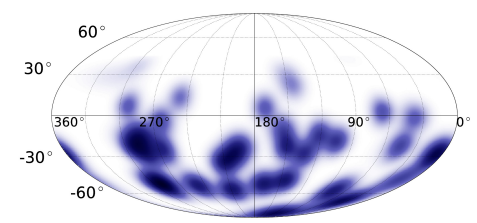

(a)

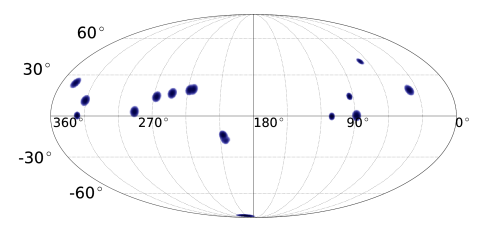

(c)

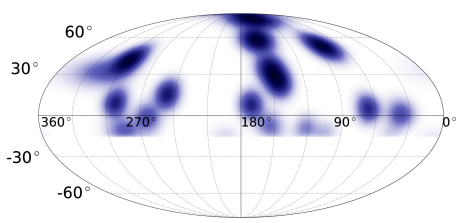

(b)

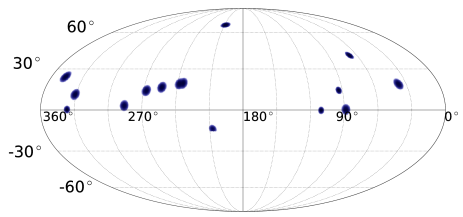

(d)

Figure 4: The signal PDFs before the Gaussian smearing in equatorial coordinates. The upper plots are for the high-energy cascades, while the lower ones are for the high-energy tracks. The declination-dependent exposure is applied for Auger in the left-hand plots and for TA in the right-hand plots.

\begin{tabular}{lllll}
\hline$D$ & \multicolumn{2}{c}{ High-energy tracks } & \multicolumn{2}{c}{ High-energy cascades } \\
& $n_{\mathrm{s}}$ & pre-trial $p$-value & $n_{\mathrm{s}}$ & pre-trial $p$-value \\
\hline $3^{\circ}$ & 4.3 & 0.22 & 53.7 & $2.1 \times 10^{-3}$ \\
$6^{\circ}$ & 0.5 & 0.48 & 85.7 & $2.7 \times 10^{-4}$ \\
$9^{\circ}$ & - & underfluctuation & 106.1 & $3.8 \times 10^{-4}$ \\
\hline
\end{tabular}

Table 1: Results for the stacking analyses with the sample of high-energy tracks and high-energy cascades.

cascades. The observed pre-trial $p$-value of $2.7 \times 10^{-4}$ corresponds to $8 \times 10^{-4}$ post-trial, i.e. after accounting for the 3 values of $D$ considered. Therefore, we obtain a potentially interesting result with the cascades as in the case of the cross-correlation analysis, which will be further studied with a larger number of events.

The angular distance at which an excess would occur in the case of the cross-correlation includes not only the magnetic deflections at the corresponding $\mathrm{CR}$ energies but also the experimental angular uncertainties. In the case of cascades, the angular uncertainty is $\sim 15^{\circ}$ and it is $\sim 1^{\circ}$ for CRs. Since most CRs in the data set have $E_{\mathrm{CR}} \sim 60 \mathrm{EeV}$, the assumed magnetic deflection where the smallest $p$-value is found in the case of the likelihood analysis with the cascades $\left(\sigma_{\mathrm{MD}}\left(E_{\mathrm{CR}}\right)=6 \times 100 \mathrm{EeV} / E_{\mathrm{CR}}\right)$ is $\sim 10^{\circ}$ in most cases. To translate this into an angular scale where one would find an excess in the cross-correlation analysis (if there were a signal), we add in quadrature and we obtain $\sqrt{\left(15^{\circ}\right)^{2}+\left(1^{\circ}\right)^{2}+\left(10^{\circ}\right)^{2}} \sim 18^{\circ}$. This scale is comparable to the $22^{\circ}$ where the smallest $p$-value is found for the cross-correlation performed with the cascades. Hence, the magnetic deflection of the CRs one would infer from the cross-correlation analysis with the cascades is comparable to the one assumed for the smallest $p$-value in the likelihood analysis, even if none of the results are at a level where no strong claims can be made.

\subsection{Stacking search for neutrino point-sources in the 4 year point-source sample}

The $v$ s data set used for this analysis is the IceCube point-source data set. A stacking analysis 
is done but in this case (as opposed to the previous one) the stacked sources are the measured positions of UHECRs. An unbinned likelihood method is performed where the log likelihood is defined as: $\ln \mathscr{L}\left(n_{\mathrm{s}_{v}}, \gamma\right)=\sum_{i=1}^{N_{v}} \ln \left(\frac{n_{s_{v}}}{N_{v}} S_{i}\left(\gamma, E_{i}\right)+\left(1-\frac{n_{s_{v}}}{N_{v}}\right) B_{i}\right)$, with $n_{\mathrm{s}_{v}}$ the total number of neutrino signal events and $\gamma$ the neutrino spectral index assuming a power-law energy spectrum. The stacked signal PDF is defined as $S_{i}=\sum_{j=1}^{N_{C R}} R_{\mathrm{IC}}\left(\delta_{j}, \gamma\right) S_{i}^{j} / \sum_{j=1}^{N_{C R}} R_{\mathrm{IC}}\left(\delta_{j}, \gamma\right)$, with $R_{\mathrm{IC}}\left(\delta_{j}, \gamma\right)$ the IceCube acceptance at the declination of a CR $j$. The signal PDF is $S_{i}^{j}=\frac{1}{2 \pi\left(\sigma_{i}^{2}+\sigma_{j}^{2}\right)} e^{-r_{i j}^{2} / 2\left(\sigma_{i}^{2}+\sigma_{j}^{2}\right)} P\left(E_{i} \mid \gamma\right)$, where $r_{i j}$ is the angular distance between the $v$ s and CRs, $\sigma_{i}$ is the angular resolution of the $v$ and $P\left(E_{i} \mid \gamma\right)$ is the energy PDF (function of the reconstructed energy proxy $E_{i}$ and $\gamma$ ). The CR deflection is modeled as an extension of the source in the likelihood with $\sigma_{j}=\sqrt{\sigma_{\mathrm{MD}}^{2}+\sigma_{\mathrm{exp}}^{2}}$, where $\sigma_{\exp }=0.9^{\circ}$ or $1.5^{\circ}$ is the experimental angular resolution of Auger or TA, respectively. The background PDF is $B_{i}=B\left(\theta_{i}\right) P_{\text {atm }}\left(E_{i}\right)$ where the energy PDF $P_{\text {atm }}\left(E_{i}\right)$ represents the probability of obtaining an energy $E_{i}$ from atmospheric backgrounds. The two free parameters are $n_{\mathrm{s}_{v}}$ and $\gamma$.

If we were to consider the entire data set of UHECRs as sources in the likelihood, their total extensions would cover a considerable amount of the sky, reducing the effectiveness of the anisotropy search. Hence we decided to introduce a threshold energy, $E_{\mathrm{th}}$, below which the CRs would not be considered. To obtain $E_{\text {th }}$, we have performed simulations of $v$ arrival directions and we have used the real sample of UHECRs, sampling different $E_{\mathrm{th}}$ energies. The flux required for a pre-trial $p$-value of $5 \sigma$ as a function of $E_{\mathrm{th}}$, is shown in Fig. 5. With the objective of keeping the flux required per source for discovery low while keeping as many UHECR events as possible, an energy threshold $E_{\mathrm{th}}=85 \mathrm{EeV}$ has been adopted. After the application of this cut, $15 \mathrm{CRs}$ in the Northern sky and 12 CRs in the Southern sky remain. Due to the different energy ranges between the neutrino candidate events in the Southern hemisphere $(\sim 100 \mathrm{TeV}-100 \mathrm{PeV})$ and in the Northern hemisphere ( $\sim 1 \mathrm{TeV}-1 \mathrm{PeV})$, for the same number of signal events per source, the normalization of flux required for Northern sources is smaller than for Southern ones. Consequently (and thanks to the gain given from having more stacked sources), the all sky sensitivity is similar to the Northern one (Fig. 5). We have thus not made a distinction between the neutrino sets from each hemisphere in this analysis.

Applying the method to the actual data, all observations are found to be compatible with the background only hypothesis. The smallest post-trial $p$-value is $25 \%$ for the hypothesis of $D=3^{\circ}$, with a fitted excess of $\sim 123$ events and $\gamma=-3.24$. The analysis with $D=6^{\circ}$ yields a $p$-value larger than $50 \%$.

\section{Conclusions}

Three analyses have been performed to investigate correlations between UHECRs detected by the Pierre Auger Observatory and Telescope Array with various samples of IceCube $v$ candidates. The results we obtained are all below $3.3 \sigma$. There is a potentially interesting result in the analyses performed with the set of high-energy cascades when comparing the results to isotropic arrival directions of CRs. If we compare the results to an isotropic flux of neutrinos (fixing the positions of the CRs) to consider the effect of anisotropies in the arrival directions of CRs (such as the TA hot spot), the significance is $\sim 2.4 \sigma$. These results were obtained with relatively few events and we will update these analyses in the future with further statistics to follow their evolution. 


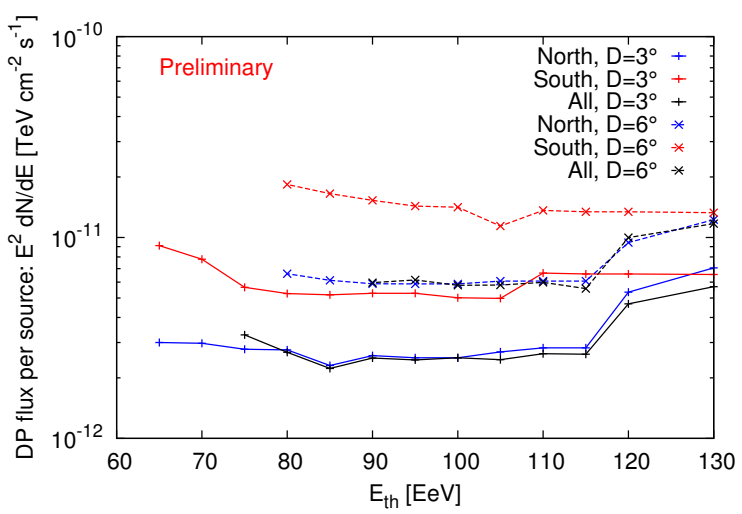

Figure 5: Flux normalization required per source for a discovery potential (DP) of $5 \sigma$ (pre-trial) for the stacking analysis with the neutrino point-source data set and UHECRs with energies above values of $E_{\text {th }}$, for the Northern and Southern skies separately and together, for the assumed deflections $\sigma_{\mathrm{MD}}\left(E_{\mathrm{CR}}\right)=D \times$ $100 \mathrm{EeV} / E_{\mathrm{CR}}, D=3^{\circ}, 6^{\circ}$.

\section{References}

[1] The IceCube Collaboration, Astropart. Phys. 26 (2006) 155.

[2] The IceCube Collaboration, JINST 9 (2014) P03009.

[3] The IceCube Collaboration, Science 342 (2013) 1242856, Phys. Rev. Lett. 113 (2014) 101101 and PoS(ICRC2015) 1081 these proceedings.

[4] The IceCube Collaboration, submitted to Phys. Rev. Lett. [arXiv:1507.04005].

[5] The IceCube Collaboration, Astrophys. J. 796 (2014) 109.

[6] The Pierre Auger Collaboration, accepted for publication in Nucl. Instrum. Meth. A (2015) [arXiv:1502.01323].

[7] The Pierre Auger Collaboration, Astrophys. J. 804 (2015) 1.

[8] C. Bonifazi for the Pierre Auger Collaboration, Nucl. Phys. B (Proc. Suppl.) 190 (2009) 20.

[9] The Pierre Auger Collaboration, JCAP 8 (2014) 19; R. Pesce for the Pierre Auger Collaboration, Proc. 32nd ICRC, Beijing, China, 2 (2011) 214 [arXiv:1107.4809].

[10] The Telescope Array Collaboration, Nucl. Instrum. Meth. A 689 (2012) 87 and Nucl. Instrum. Meth. A 676 (2012) 54.

[11] The Telescope Array Collaboration, Astrophys. J. Lett. 790 (2014) L21.

[12] The Telescope Array Collaboration, Astropart. Phys. 48 (2013) 16.

[13] M. S. Pshirkov, P. G. Tinyakov, P. P. Kronberg, K. J. Newton-McGee, Astrophys. J. 738 (2011) 192.

[14] R. Jansson and G. R. Farrar, Astrophys. J. 757 (2012) 14.

[15] J. Braun, J. Dumm, F. De Palma, C. Finley, A. Karle, T. Montaruli, Astropart. Phys. 29 (2008) 299. 